\title{
A feasibility study on different NDT techniques used for testing bond quality in cold roll bonded Al-Sn alloy/steel bimetal strips
}

\author{
Philipp Johannes Tallafuss ${ }^{1,2, *}$, Andrzej Rosochowski ${ }^{1}$, and Sylvia Campbell ${ }^{2}$ \\ 1 Department of Design, Manufacture and Engineering Management (DMEM), University of Strathclyde, Glasgow, UK \\ 2 MAHLE Engine Systems and Components UK Ltd., Glasgow, UK
}

Received 19 December 2016 / Accepted 11 March 2017

\begin{abstract}
This paper presents non-destructive testing (NDT) results for the detection of bond defects in aluminiumtin (Al-Sn) alloy/steel bimetal strips. Among all types of bimetal strip that are used in the automotive industry for plain journal engine bearings, Al-Sn alloys cold roll bonded (CRB) onto steel backing is the most common type. The difficulty to evaluate the metallurgical bond between the two dissimilar metals is a major industrial concern, which comprises the risk that bearings fail in the field. Considering the harsh performance requirements, $100 \%$ online non-destructive testing would be desirable to significantly reduce the business risk. Nowadays bimetal strip manufacturers still rely on destructive testing through different peel-off tests. This work offers the results from four independent NDT studies, using active thermography, shearography, ultrasound and guided wave electromagnetic acoustic transducers (EMATs) and samples with different artificially implanted defects, to explore the feasibility to qualitatively indicate the occurrence of bond defects. A destructive peel off test was used to correlate the NDT results with known bond quality. The studies were done under laboratory conditions, and in case of ultrasound also online under production conditions. During the ultrasound online test, the requirements that a NDT technique has to fulfil for online inspection of Al-Sn alloy/steel bimetal strip were established. For active thermography, shearography and guided wave EMAT techniques, it was theoretically analysed, if the laboratory test results could be transferred to testing under production conditions. As a result, guided waves using EMATs, among the four tested methods, are best suited for online inspection of Al-Sn alloy/steel bimetal strip. This research was carried out in collaboration with MAHLE Engine Systems UK Ltd., an Al-Sn alloy/steel bimetal strip manufacturer for the automotive industry.
\end{abstract}

Key words: Cold roll bonding, Non-destructive testing, Bond defects, Bimetal strips

\section{Introduction}

Al-Sn alloy/steel bimetal strips are used for engine bearings in the automotive industry due to the ideal hybrid properties of Al-Sn alloy and steel [1]. The properties are wear and corrosion resistance as well as high anti-seizure properties [2]. The aluminium alloy that is in sliding contact between the surfaces has embedability, conformability and compatibility properties, which are achieved by adding tin as an alloying element [3]. This is required to resist welding between the sliding surfaces, allow clearance under the heat, pressure and foreign particles that are present in an engine.

Clad aluminium-steel products are either manufactured by roll bonding, explosion welding or fusing [4]. This work is based on bimetal strips for which the clad $\mathrm{Al}$ is cold roll bonded (CRB) to steel. In CRB the solid state weld is achieved by a substantial and simultaneous plastic deformation of the

*e-mail: philipp.tallafuss@gb.mahle.com metals at room temperature [1]. Figure 1 illustrates the CRB process schematically.

The disadvantage of this technique is the large number of secondary operations and the high requirements for the bonding surface preparation quality [4]. In the MAHLE Kilmarnock Scotland plant, the internal Process Failure Mode Effects Analysis (PFMEA) of their continuous CRB production line, identified 171 different root causes for poor bond in the secondary and surface preparation operations. Considering the difficulty to create a metallurgical bond between two dissimilar metals in the CRB process and the limitations of destructive testing, there is a need for an automated online non-destructive testing (NDT) system that is capable of inspecting the bond of Al-Sn alloy/steel bimetal strips during production. This would significantly reduce the risk for engine bearing manufacturers that bearings fail in the field.

Figure 2 shows a micro section of the four-layered $\mathrm{Al} / \mathrm{Al}-\mathrm{Sn} / \mathrm{Al} /$ steel composite structure. The surface cladding layers of the Al-Sn internal core are made of Al1050 strip. 


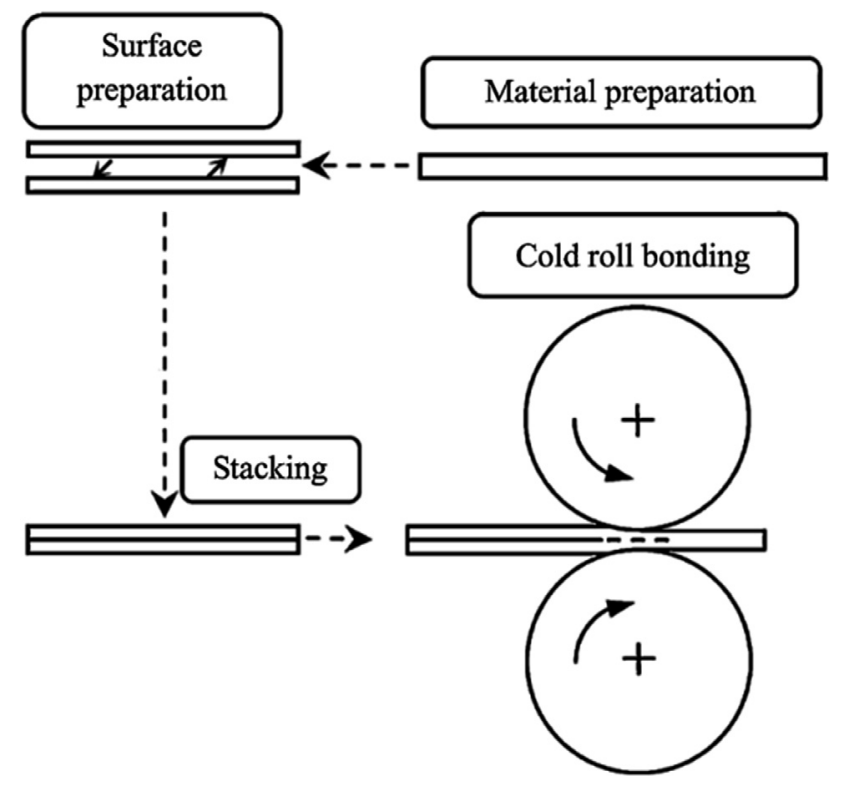

Figure 1. Cold roll bonding (CRB) schematic process [5].

The Al-Sn alloy has a nominal $20 \%$ tin content. The backing is made of a low carbon steel. The clad Al/Al-Sn/Al is cold roll bonded onto the steel free from aligned linear porosity or continuous oxides. The bimetal strip thickness ranges from 1.25 to $4.25 \mathrm{~mm}$ with a minimum thickness of $0.51 \mathrm{~mm}$ of the clad $\mathrm{Al}$ layer.

The material properties that are required to determine the inspection parameters for the various NDT techniques are listed in Table 1. The material grains, which are normally equiaxial in the isotropic state, become elongated and flattened during rolling. All study samples were annealed after rolling to recrystallize the clad $\mathrm{Al}$ grain structure and obtain quasi equiaxed grains post rolling anneal, before the samples were inspected. Any remaining anisotropy post rolling annealing was neglected in the study.

NDT of composite materials is becoming increasingly attractive in the automotive industry as a requirement for more energy-efficient vehicles [6]. NDT of the bond integrity of multi-layered structures is of interest and of practical value, which is why a large body of research literature has been published on the subject. Traditional ultrasound longitudinal and shear bulk waves [7-9], as well as more recently Lamb or Shear-Horizontal $(\mathrm{SH})$ guided waves [10-12] are ultrasound techniques that get increasingly applied to inspect the bond of multi-layered bonds. Shearography [13-15] and Thermography [15-18] are innovative optical techniques that allow full-field, non-contact and speedy detection in composite materials. However, no research papers were published on the application of these NDT techniques to Al-Sn alloy/steel bimetal strip that was manufactured in the CRB process. Furthermore to date no NDT technique is commercially available for the bond inspection of CRB of Al-Sn alloys to steel, although the majority of bimetal bearings in automotive engines are made of this material. The purpose of this work is to further understand the requirements that a NDT testing technique has to fulfil to be suitable for online inspection in a serial production

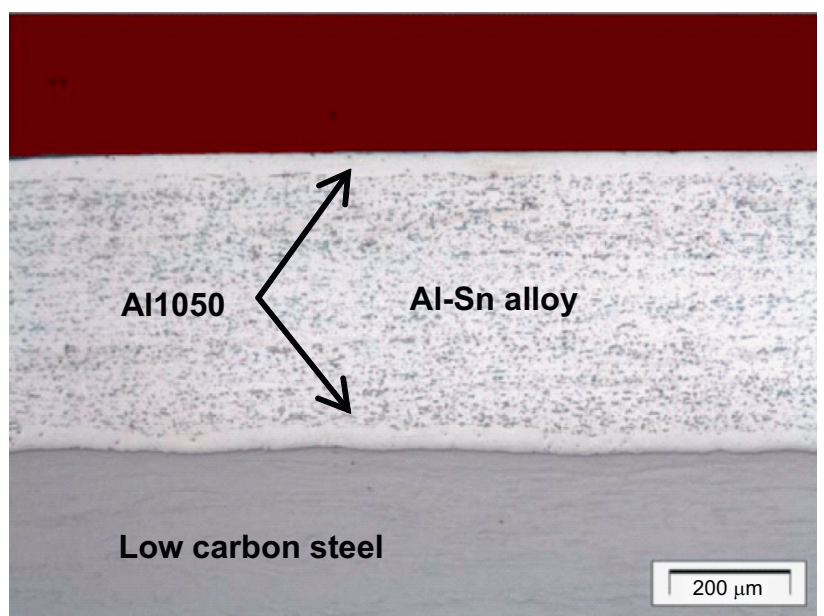

Figure 2. Cross section view of the four-layered composite structure.

Table 1. Material properties for bimetal strip.

\begin{tabular}{lcccc}
\hline \multicolumn{1}{c}{ Characteristics } & Layer 1 & Layer 2 & Layer 3 & Layer 4 \\
\hline Material & Al1050 & Al-Sn alloy & Al1050 & Steel \\
Density $\left(\mathrm{g} / \mathrm{cm}^{3}\right)$ & 2.71 & 3.11 & 2.71 & 7.8 \\
Young's modulus $(\mathrm{GPa})$ & 70 & 70 & 70 & 203 \\
Poison's ratio & 0.33 & 0.33 & 0.33 & 0.293 \\
\hline
\end{tabular}

environment. The available literature on NDT of composite materials was used to identify potential NDT techniques. A peel off test, which is one of the destructive tests in industry that is currently used by the bimetal strip manufacturers as bond test during production, was used to correlate the sample NDT results with destructive testing results.

\section{Destructive and non-destructive testing methods}

\subsection{Clad Al lining adhesion peel back test}

Current industry practice for Al-Sn alloy/steel bimetal strip bond inspection is destructive testing using a chisel test, peel test, shear test, Erichsen cup test or hot hammer test [13]. Although each technique has specific advantages and disadvantages, they all have in common that only a minor proportion of the produced material is inspected.

The bond peel back test to assess the adhesion between clad Al lining and steel backing is one of the most established tests in the strip manufacturing industry. Therefore this test is used in this study to obtain pass/fail measurement data for the test samples.

The test procedure is illustrated in Figure 3. At first, an incision through the lining is made with a sharp chisel until the chisel strikes the steel backing. The clad Al lining is then prised upwards until the material can be gripped with a pair of round nosed pincers. The lining is peeled back from the steel in a rotational movement. When the peel back area shows a clean 




Figure 3. (a) Peel back of alloy using round nosed pincers, (b) pass/fail ratings for the peel back test.

steel surface without any clad Al lining material, then the alloy to Al1050 bond is stronger than the Al1050 foil to steel bond. Fracture of the clad Al lining that would give an inaccurate test result, is prevented by continually re-gripping the lining throughout the test. The length of the lining peel back prior to fracture is measured and compared against the acceptability ratings shown in Figure 3. The acceptability ratings were established with empirical test data.

In serial production, this test is carried out for each sample at a minimum of five positions across the width of the Al-Sn alloy/steel bimetal strip. The test samples in this study were tested at the known locations of the artificially implanted defects to obtain a peel back result. Despite limitations of this destructive testing technique, such as limited reproducibility and repeatability because of the human influencing factor, the quality assurance of this technique is time-proven in industry and therefore plays an important role in the development of a NDT technique for serial inspection of Al-Sn alloy/steel bimetal strips.

\subsection{Thermography}

Temperature is one of the most common indicators of the structural health of components. Typical examples are damaged machineries, corroded electrical connections and damaged material components, which cause an abnormal temperature distribution. Recently thermography became a widely accepted NDT technique in industry, which uses the abnormal temperature distribution to detect flaws [19].

Thermography is widely used to inspect buildings and civil structures [20], in the automotive and aerospace industry in numerous applications for flaw detection, bonding integrity assessment and composite structure evaluation [16, 17, 21], and diverse other applications such as pressure vessel evaluation [22], pipeline inspection [23] and micro-crack inspection [24]. The transient pulse heating technique has proven to be the most suitable method for bond integrity testing for adhesive bonds, e.g. for panel frame structures or the bonnet of a car, because the adhesive bond is usually close to the surface of the material [15]. This is the reason for selecting the transient pulse thermography technique for the bond inspection of Al-Sn alloy/steel bimetal sheets, in which the clad Al to steel delamination is close to the surface of the sample.

The functional principle in active thermography is that heat is transferred into the material that causes an instantaneous thermal excitation and the materials' heat-transfer response is measured. It is a fast technique allowing real-time inspection, provides $100 \%$ check and is a non-contact technique to inspect the material [15]. Flaws are revealed by searching anomalous hot-spots that are caused by the flaws after thermal excitation. As thermography is a thermal radiation measurement technique, flaws cause local variations in the observed surface temperature pattern [15]. While in passive thermography only the variation of surface radiation is measured, a controllable thermal source to excite the material and reduce environmental influences is used in active thermography to inspect the material. Common active thermography techniques are transient pulse, step heating, periodic heating as well as thermal mechanical vibration [15]. The harsh production environment of Al-Sn alloy/steel bimetal strips with varying ambient conditions (vibrations, temperature, and light conditions) is a challenge to apply shearography for online inspection. However, recent developments such as the Thermographic Signal Reconstruction (TSR) method can enhance the analysis of thermographic sequences by creating a noise reduced replica of each pixel, which allows improved analysis over unprocessed image data [25].

\subsection{Shearography}

Shearography is a technique that is already well established in industry for NDT [13]. It has been successfully employed for adhesive bonding integrity inspection for multi-layered composites, e.g. GLARE panels, honeycomb structures and glass (or carbon)-fibre-reinforced plactics [26]. Other shearography applications are measurement of specific surface strains, residual stresses, 3D shapes and vibration modes, and leakage detection [14]. All these applications have in common, that the samples are usually static.

Bond defects in composites are detected by applying a known external excitation that leads to deformation of the material, which enables NDT of the samples as the basic principle of this technique is based upon the response of the flaw to the applied stress. The techniques include: pulse thermography [27], lock-in thermography [28], thermosonics [29], and digital shearography [30]. Digital shearography has a broad application field to provide information about flaws in materials such as discontinuities and separations, dimensions, as well as stress and dynamic response. Displacement and displacement-derivatives are considered to measure the surface deformation. Flaws are revealed by comparing flawless deformation areas to flaw containing deformation anomalies, after the material is stressed. Analysis of the fringe patterns and backward calculations are used to detect the flaw. Typical methods for material deformation that are non-contact and cause small rigid-body movement are pressurization, partial vacuum, acoustics as well as thermalshock excitation [15]. In an ideal case, the applied stress is similar to the service stress. This way only flaws that are critical in service are detected and cosmetic flaws are ignored [13]. 
Furthermore it is a fast technique allowing real-time inspection, provides $100 \%$ check and is a non-contact technique to inspect the material [15].

A limitation for online NDT using shearography is the presence of higher disturbances, especially vibrations, which introduces unknown phase deviations in the shearographic images, which makes bond defect detection impossible. Recently a new approach to improve the quality of shearographic phase maps that are acquired in a harsh environment was presented, which combines the N-dimensional Lissajous algorithm and the concept of phase of differences, to improve the quality of phase maps [31]. This progress could facilitate the application of digital shearography for the online inspection of Al-Sn alloy/steel bimetal strip.

\subsection{Ultrasound}

Ultrasonic testing is considered as the most efficient inspection method in all major industries, such as electrical and electronic components manufacturing, metallic and composite material production, structures fabrication like airframes, piping and pressure vessels, ships, bridges, motor vehicles, machinery and jet engines [32]. Ultrasonic testing methods are the most applied methods in non-destructive testing of multi-layered composites [33].

In ultrasound inspection, sonic energy is used to locate and identify surface and subsurface flaws. In the ASM handbook [34], the pulse-echo method is presented as the most widely used configuration, in which the ultrasound pulse is reflected from a discontinuity. The functional principle is that waves travel in a straight line and at a constant speed until they encounter an anomaly in the material, which reflects the ultrasound wave. The reflected signals from the flaw surface that the sound beam encounters are differently attenuated. Flaws such as cracks, holes, delaminations, slags, cavities, porosities, bursts, lack of fusion or flakes that have sharp boundaries, are easily detected and identified by ultrasonic testing [35].

A typical ultrasound amplitude representation technique is a C-scan, in which an attenuation map of the scanned area of the part is shown. The flaw depth, which is dependent on the ultrasound time of flight, is usually not recorded, but can be controlled accurately by setting inspection gates. A limitation of $\mathrm{C}$-scans is that the interpretation can be misleading due to noise; however the development and use of advanced transducers and the advancement in digital signal processing improved the effectiveness of using ultrasonic testing for composite materials [33].

Piezoelectric ultrasound transducers require a coupling media to transmit the ultrasonic sound from the probe into the material to prevent an air gap with significant impedance mismatch between the probe and the material. Coupling is either achieved by immersion testing, typically for smaller test specimens, or by using water jet probes for larger components [36]. This coupling media requirement can restrict ultrasound inspection for certain applications, in which no coupling media can be applied on the sample. A limitation of ultrasound is that it requires a two-dimensional scan to cover the entire material volume, which makes it difficult to inspect material online at high speed [37].

\subsection{Guided waves using EMATs}

Electromagnetic acoustic transducers (EMATs) belong to the group of non-contact ultrasonic transducers [38]. Typical applications are the inspection of pipes to detect corrosion or cracks [39], the condition of rails [40], weld defect detection [41], adhesive bond inspection in composites [42] and metal plates [37, 43, 44].

EMATs are capable to emit and receive ultrasound on conductive metals, and they are in particular suitable when the application requires a contactless inspection. Typical cases are, when the sample is hot, moving, or otherwise not suitable for a transducer to be directly in contact with the material, or when no coupling media or surface preparations are possible to use [44-46]. Most of these criteria apply for the online inspection of Al-Sn alloy/steel bimetal, which is moving, contact should be avoided to not damage the surface, and carry over of the coupling media into the material is not desired. Even though EMAT is in theory a contactless technique, close proximity is required. The maximum possible lift-off between the EMAT probe and material depends on the EMAT design, material properties, and the acoustic wave frequency [47]. Studies report that due to the requirement of a reasonable signal-to-noise ratio (SNR), the lift-off is typically restricted to below $3 \mathrm{~mm}$ [48].

EMAT sensors typically have a built in neodymium-ironboron $\left(\mathrm{Nd}_{2} \mathrm{Fe}_{14} \mathrm{~B}\right)$ sintered permanent magnet that provides a static magnetic field, and a coil of wire to induce eddy currents at the skin depth of the sample. The ultrasound generation and reception between sample and probe is an electromagnetic mechanism due to the Lorentz force, and can involve magnetostriction, as well as the magnetisation force between the oscillating magnetic field of the probe and the static magnetic field of the ferromagnetic material [49-51].

EMATs are relatively inexpensive to produce, however they are less efficient at generating or detecting ultrasound compared to piezoelectric transducers [52]. An advantage compared to the pulse-echo or through transmission ultrasound technique is that a conventional two-dimensional scan is reduced to a one-dimensional scan, which increases inspection speed [37]. Furthermore guided waves that can be generated using EMATs are well suited for thin plate inspection. The technique was already successfully applied in the inspection of three-layered clad of brass/copper/brass material for coin stock [37]. A recent development in the guided wave inspection of metallic plates is, to attempt to accurately determine the size and shape of defects instead of only detecting the defects, which is possible by using an array of transducers to transmit and receive guided waves from all directions [43].

\section{Experimental procedures}

\subsection{Thermography}

\subsubsection{Sample preparation}

Table 2 summarises the samples that were used in the active thermography feasibility study. Sample AT-R is produced under 
Table 2. Test samples in relation to the applied NDT methods.

\begin{tabular}{|c|c|c|c|c|c|c|c|c|c|}
\hline$\#$ & $\begin{array}{c}\text { NDT } \\
\text { technique }\end{array}$ & Identity & $\begin{array}{l}\text { Nominal } \\
\text { compound }\end{array}$ & $\begin{array}{c}\text { Clad Al } \\
\text { thickness } \\
(\mathrm{mm})\end{array}$ & $\begin{array}{c}\text { Steel } \\
\text { thickness } \\
(\mathrm{mm})\end{array}$ & $\begin{array}{l}\text { Red } \\
(\%)\end{array}$ & $\begin{array}{c}\text { Sheet } \\
\text { dimension } \\
(\mathrm{mm})\end{array}$ & Surface preparation & $\begin{array}{l}\text { Peel } \\
\text { back } \\
(\mathrm{mm}) \\
\end{array}$ \\
\hline 1 & $\begin{array}{l}\text { Active } \\
\text { thermography }\end{array}$ & AT-R & AlSn20CuMn & 0.67 & 1.96 & 43 & $280 \times 171 \times 2.63$ & Reference & $\overline{\text { No peel }}$ \\
\hline 2 & $\begin{array}{l}\text { Active } \\
\text { thermography }\end{array}$ & AT-NL & AlSn20CuMn & 0.52 & 2.13 & 43 & $280 \times 171 \times 2.65$ & No linishing & 13 \\
\hline 3 & $\begin{array}{l}\text { Active } \\
\text { thermography }\end{array}$ & AT-RR & $\mathrm{A} 1 \mathrm{Sn} 20 \mathrm{CuMn}$ & 0.63 & 2.00 & 30 & $280 \times 171 \times 2.63$ & $\begin{array}{l}\text { Rolling reduction } \\
\quad 30 \%\end{array}$ & 9 \\
\hline 5 & Shearography & S-SP & AlSn20CuMn & 0.53 & 1.22 & 43 & $280 \times 171 \times 1.75$ & $80 \mathrm{~g} / \mathrm{m}^{2}$ sheet paper & $>5$ \\
\hline 6 & Ultrasonic testing & UT-R & AlSn20CuMn & 0.53 & 1.22 & 43 & $280 \times 171 \times 1.75$ & Reference sample & No peel \\
\hline 7 & Ultrasonic testing & UT-NB & AlSn20CuMn & 0.53 & 1.22 & 43 & $280 \times 171 \times 1.75$ & No brushing & 2 \\
\hline 8 & Guided wave EMAT & GW-R & A1Sn20CuMn & 0.53 & 1.22 & 43 & $800 \times 171 \times 1.75$ & Reference sample & No peel \\
\hline 9 & Guided wave EMAT & GW-PT & $\mathrm{AlSn} 20 \mathrm{CuMn}$ & 0.53 & 1.22 & 43 & $800 \times 171 \times 1.75$ & $\begin{array}{l}80 \mathrm{~g} / \mathrm{m}^{2} \text { paper } \\
\text { triangle }\end{array}$ & $>5$ \\
\hline
\end{tabular}

normal parameters and is used as reference sample. The peeloff test confirmed sound Al1050 foil to steel bond as there was no peel back in the test that was done according the procedure described in Section 2.1. This sample is made of $0.67 \mathrm{~mm}$ thick clad $\mathrm{Al}$ and $1.96 \mathrm{~mm}$ thick steel sheets after $43 \%$ thickness reduction in CRB. The sample dimensions are $280 \mathrm{~mm}$ long and $171 \mathrm{~mm}$ wide and have a total thickness of $2.63 \mathrm{~mm}$.

For the AT-NL sample no linishing was applied to the steel surface. Various studies [53, 54], reported that steel linishing is essential to establish an effective cold weld during CRB because it removes contaminants and surface oxides that could interfere with the creation of nascent metal. Furthermore it has been reported that localised shear deformation to break unavoidable surface oxide films is promoted by a larger amount of surface asperities, which are created by steel surface linishing [55]. MAHLE plant trials substantiate that there is a negative effect on the bond integrity, if the steel surface is not linished before CRB. The AT-NL sample prepared in this specific way failed the peel back test, as the peel back was about $13 \mathrm{~mm}$. This sample is made of $0.52 \mathrm{~mm}$ thick clad $\mathrm{Al}$ and $2.13 \mathrm{~mm}$ thick steel sheets after $43 \%$ thickness reduction in CRB. The sample dimensions are $280 \mathrm{~mm}$ long and $171 \mathrm{~mm}$ wide and have a total thickness of $2.65 \mathrm{~mm}$.

The same process is used for sample AT-RR as for sample AT-R, except for the thickness reduction during CRB which was only $30 \%$ instead of the standard $43 \%$ thickness reduction. The thickness reduction in bimetal CRB is the most influencing factor on the bond strength [56]. This is because of the direct relation between thickness reduction, surface expansion and extrusion of virgin metal [57]. The AT-RR sample prepared in this specific way also failed the peel back test, since the peel back was about $9 \mathrm{~mm}$. This sample is made of $0.63 \mathrm{~mm}$ thick clad $\mathrm{Al}$ and $2.00 \mathrm{~mm}$ thick steel sheets after $30 \%$ thickness reduction in CRB. The sample dimensions are $280 \mathrm{~mm}$ long and $171 \mathrm{~mm}$ wide and have a total thickness of $2.63 \mathrm{~mm}$.

\subsubsection{Experimental set up}

Figure 4 is a schematic of the transient pulse thermography experimental setup. Thermal radiation in the reflection mode of the one-time transient pulse excitation technique was used in this experiment. This technique has the advantages of fast inspection speed, full-field interrogation, as well as being contactless. An extremely short exposure time $(\sim 3 \mathrm{~ms})$ and transient light sources with large power $(\sim 9.6 \mathrm{~kJ})$ excite the material. The samples were placed horizontally in a stable position on a measurement table throughout the measurement process to keep the bimetal samples perfectly aligned with the two flash lamps and the infrared camera. The clad Al surface is facing the lighting source to heat it up with a short and spatially uniform light pulse. Delamination areas are identified by an infrared (IR) camera that is connected to a PC, which monitors and analyses the timedependent sample surface temperature response relative to the thermal pulse.

The delaminated areas in the bimetal sample obstruct the transient heat flow from surface into the sample interior during the thermal excitation, because the vacuum between the layers acts as a lagging. This produces an increase in the local transient temperature of the alloy surface, directly above the delamination. These anomalous "hot-spots" are revealed with time. The materials' characteristic diffusivity and delamination depth that is dependent on the clad $\mathrm{Al}$ and steel sample thickness, and their influence on the transition time were taken into consideration when the model was setup. The sequential data sets are considered as independent time histories of the temperature at each pixel, which is described and analysed with a onedimensional diffusion model. In this model, the surface temperature depends only on time. Sample variables in the model are thermal conductivity, density and the specific heat. The inspection set-up variables are input energy per unit area. For a defective sample the temperature slope deviates from the characteristic slope of a defect-free sample. Focusing on the deviation of a single pixel on the surface identifies 


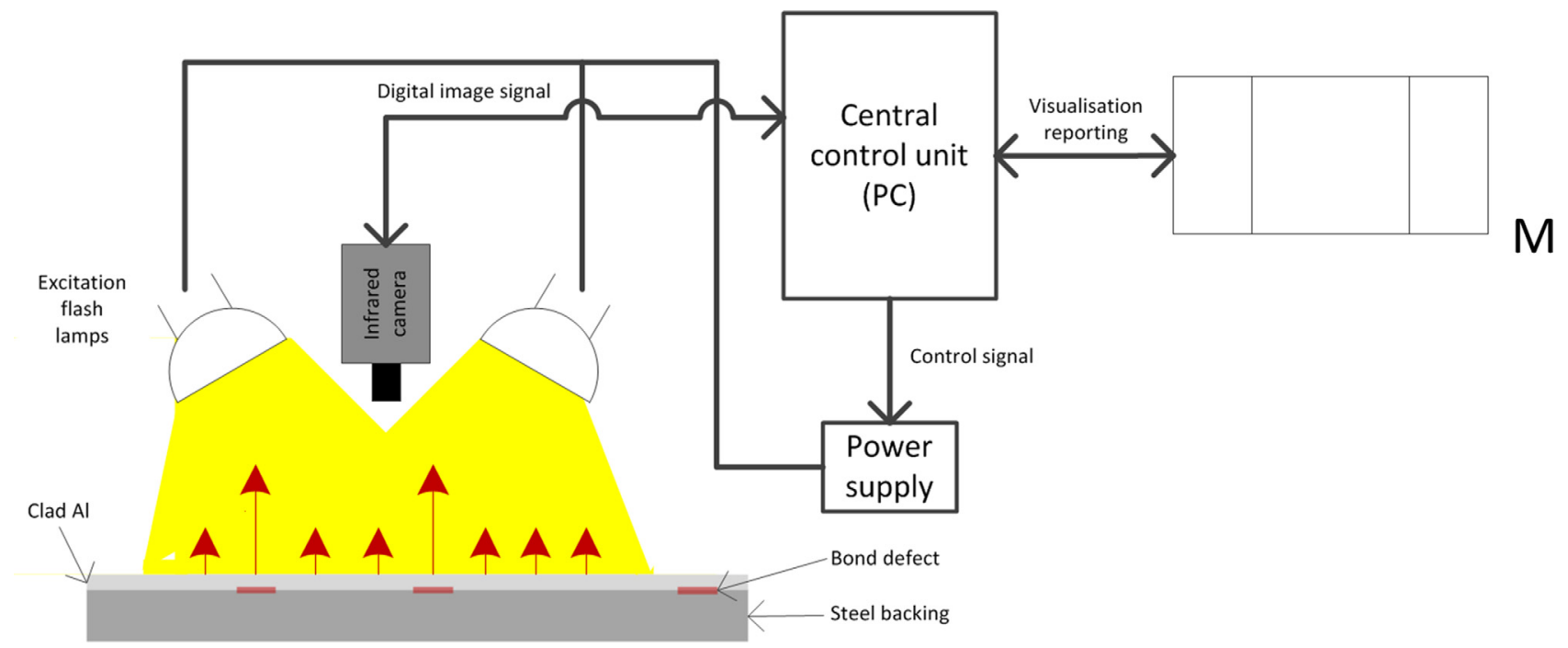

Figure 4. Schematic of experimental set up for delamination detection in transient pulse thermography.

delaminations that interrupt the heat flow without reference to the neighbours.

\subsection{Shearography}

\subsubsection{Sample preparation}

Table 2 provides an overview of the two samples that are used in the digital shearography feasibility study. Sample S$\mathrm{R}$ is the reference sample produced under normal production parameters. Good bond was confirmed with the peel-off test. S-SP is the test specimen with artificially implanted delamination, in which $140 \times 45 \mathrm{~mm} 80 \mathrm{~g} / \mathrm{m}^{2}$ gauge paper was inserted between the clad $\mathrm{Al}$ and the steel sheets before CRB. The S-SP sample failed the peel off test, since the gauge paper created a defined gap between clad Al and steel. The destructive test result was more than $5 \mathrm{~mm}$ peel off and only ended due to fracture of the clad Al. This sample preparation method is also used in other studies to create a delamination, as it does not affect the adjacent bonded interface [58]. Both samples are $280 \mathrm{~mm}$ long and $171 \mathrm{~mm}$ wide and are made of $0.53 \mathrm{~mm}$ thick clad Al and $1.22 \mathrm{~mm}$ thick steel sheets after $43 \%$ thickness reduction during CRB.

\subsubsection{Experimental set up}

Two different excitation methods, vacuum and thermal shock loading were tested in the shearography feasibility study. Vacuum loading has already been successfully tested on laboratory samples of aluminium-CFRP panels, which had artificially implanted delaminations [15]. A drawback of this technique is that it is limited to delaminations with enclosed boundaries. If the delamination in Al-Sn alloy/steel bimetal strip is at the edge of the strip, the delamination has an open boundary. Thermal shock loading overcomes this drawback and is therefore more suitable in open panels. Thermal shock excitation can be achieved by using hot air jet, heat lamp, and high power argon laser or recently developed high power
Xenon flash lamps. This technique successfully revealed flaws under laboratory conditions for subsurface cracks that were engineered in aluminium panels [15].

The feasibility testing was carried out using the Dantec Dynamics Digital Shearography NDT System Q-800. Figure 5a illustrates the thermal testing set up used. The Q-800 system was mounted on the same table as the samples, approximately $450 \mathrm{~mm}$ away from each other. A $230 \mathrm{~V} / 750 \mathrm{~W}$ heat lamp was positioned $30 \mathrm{~mm}$ away from the sample with the alloy side facing to the heat lamp. First a reference specklegram was captured without loading the sample. The illumination duration was $30 \mathrm{~s}$. Then heat energy was delivered to apply a thermal stress to the sample. Due to the thermal heat excitation, the gas inside the delamination expands, creating an internal pressure in the sample. Then another series of specklegrams was captured. Due to the temperature difference between the relatively cool interior of the specimen and the exposed surface, a thermally induced bending moment is caused. Delaminations lead to reduced local rigidity close to the delamination, which causes localized out-of-plane deformation anomalies. These anomalies were captured as fringe condensation, which reveals the delamination.

Figure $5 \mathrm{~b}$ shows the vacuum testing set up. For vacuum testing, the same Q-800 system and the samples were placed in an airtight chamber $450 \mathrm{~mm}$ apart from each other, and an air pump produced a 100 mbar partial vacuum in the chamber between the material surface and the enclosed space. As the test piece is uniformly stretched by the negative pressure difference, delamination areas will bulge and cause fringe anomaly during shearography inspection.

\subsection{Ultrasound}

\subsubsection{Sample preparation}

Two samples were manufactured for the ultrasound testing feasibility study that are summarised in Table 2. Sample UT-R is the reference sample that was produced under normal 
(a)

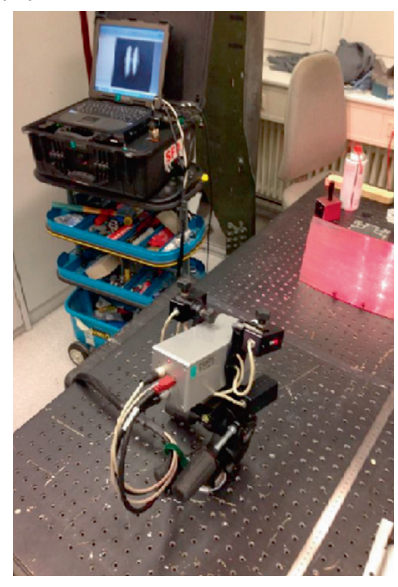

(b)

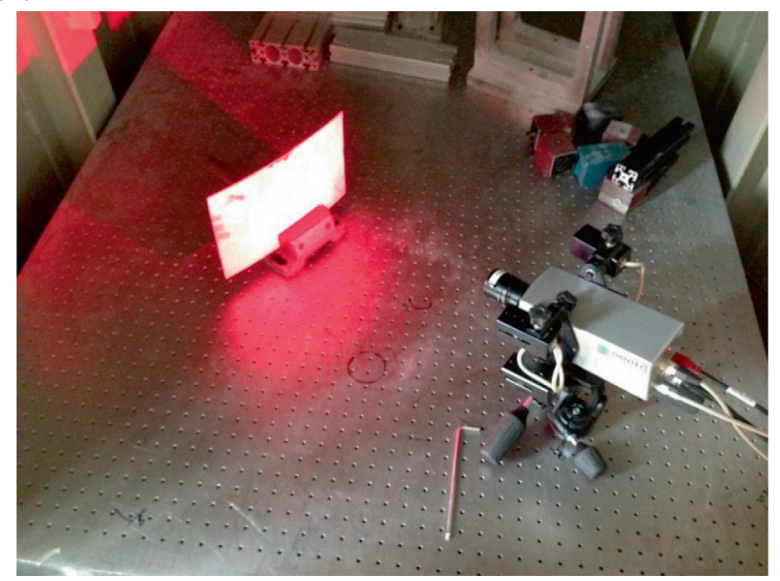

Figure 5. Shearography experimental set up for (a) thermal shock and (b) vacuum technique.

conditions. The peel off test confirmed sound Al1050 foil to steel bond, since there was no peel back in the peel back test that was done according to the procedure in Section 2.1. For the UT-NB sample, the alloy surface was not brushed prior to $\mathrm{CRB}$ to prevent an effective cold weld during CRB. It has been shown in other studies that the oxide film thickness that is present on the alloy during CRB when the alloy is not brushed prior to $\mathrm{CRB}$, does markedly decrease the bond integrity between Al1050 foil and steel [1]. Poor bond between Al1050 foil and steel for the UT-NB sample was confirmed with the peel off test, in which the peel back was about $2 \mathrm{~mm}$, which is indicating weakened bond according to the acceptability criteria in Section 2.1. The samples are $280 \mathrm{~mm}$ long and $171 \mathrm{~mm}$ wide and are made of $0.53 \mathrm{~mm}$ thick clad $\mathrm{Al}$ and $1.22 \mathrm{~mm}$ thick steel sheets after $43 \%$ thickness reduction during CRB.

\subsubsection{Laboratory experimental set up}

The pulse echo technique was used in the laboratory experiment. The system was supplied by Physical Acoustics Ltd (PAL). It had a $25 \mathrm{MHz}$ spot focused probe, stepper motor and a Data Acquisition System comprising of a $100 \mathrm{MHz}$ pulser/receiver, $1000 \mathrm{MHz} \mathrm{A} / \mathrm{D}$ converter with dual gates and a PC with Ultrawin software.

Figure 6 shows a schematic of the scanner ultrasonic inspection system set up in the laboratory. The pulse-echo scanner was submerged in the immersion tank and a stepper motor was used to scan the specimen. The ultrasonics data acquisition system was positioned next to the water tank. The system recored 100 pulses per second and a moving average of 100 pulses (1 s) was used to monitor the signal. The probe was transmitting and receiving from the steel surface and was focused onto the bond line. Two signal gates were set to receive the echo from the bond line (Gate 1) and from the alloy backwall (Gate 2). The output from the returned signal is displayed as percentage of the signal strength returned by Gate 1 divided by the signal strength returned by Gate 2 . The stronger the signal returned from the bond line is, the stronger is the reflector and consequently weaker is the bond.
Therewith the Gate 1/Gate 2 ratio is higher for weaker bonds. Figure 7 shows the $\mathrm{C}$-scan colour code that is used depending on the received signal strength. Values of Gate 1/Gate 2 ratio greater than $110 \%$ are coded red, ratio between 90 and 110 as yellow and ratio below 90 as blue.

Figure 8 shows the probe scanning pattern on the $x-z$ plane of the bimetal sample. For the pulse-echo inspection in the immersion tank only one probe is used. Scanning the material is necessary in order to cover $100 \%$ of the material. This is required as the ultrasound pulse-echo technique is by nature limited to a one dimensional measurement across the thickness normal to the plane surface of the bimetal sample.

\subsubsection{Online experimental set up}

The equipment for the online trials was the same as for the laboratory feasibility study supplied by Physical Acoustics Ltd. (PAL) with a $25 \mathrm{MHz}$ high resolution focussed ultrasonic probe. The position of the probe in the online trial was between a rolling mill exit accumulator and a coiler, where the strip movement is kept to a minimum and the steel side could be accessed. Figure 9a shows the position where the traversing scanning bridge and electronic inspection equipment was set up. Figure $9 \mathrm{~b}$ shows the bubbler head, which repeatedly traversed over a $150 \mathrm{~mm}$ width of the bimetal strip to scan the material. In order to avoid significant water carryover, a bubbler head instead of an immersion tank for the water fluid coupling medium was used for the online trial. A pump in the water container reservoir forced a column of water through the probe, which transmitted the ultrasonic signal to the steel underside of the bimetal strip. During the trials, the line ran at a constant speed of $12 \mathrm{~m} / \mathrm{min}$.

\subsection{Guided waves using EMATs}

\subsubsection{Sample preparation}

Table 2 summarises the three samples that were used for the EMAT feasibility study. Sample GW-R was manufactured 


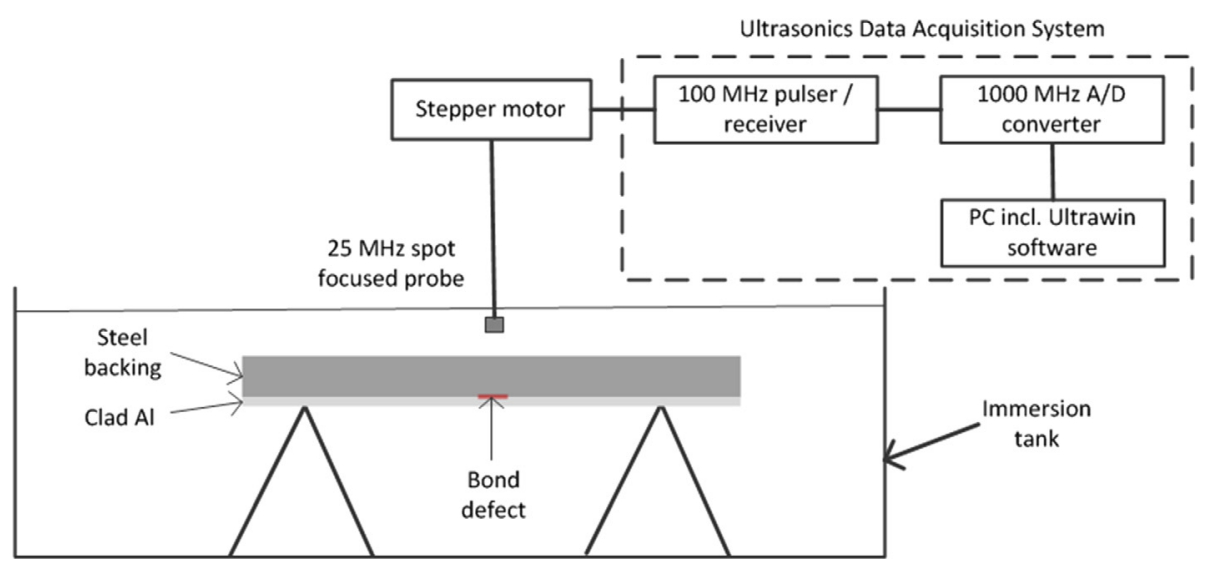

Figure 6. Schematic view of test set up showing the Al-Sn alloy/steel bimetal sample submerged in an immersion tank, stepper motor and the Data Acquisition System.

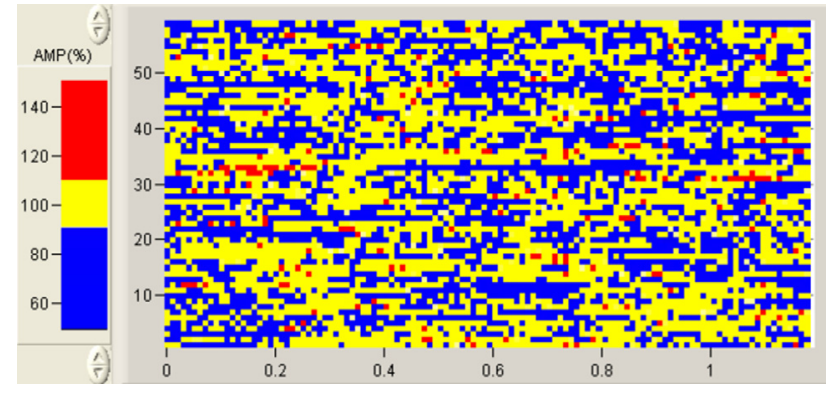

Figure 7. C-scan colour code depending on Gate $1 /$ Gate 2 amplitude ratio.

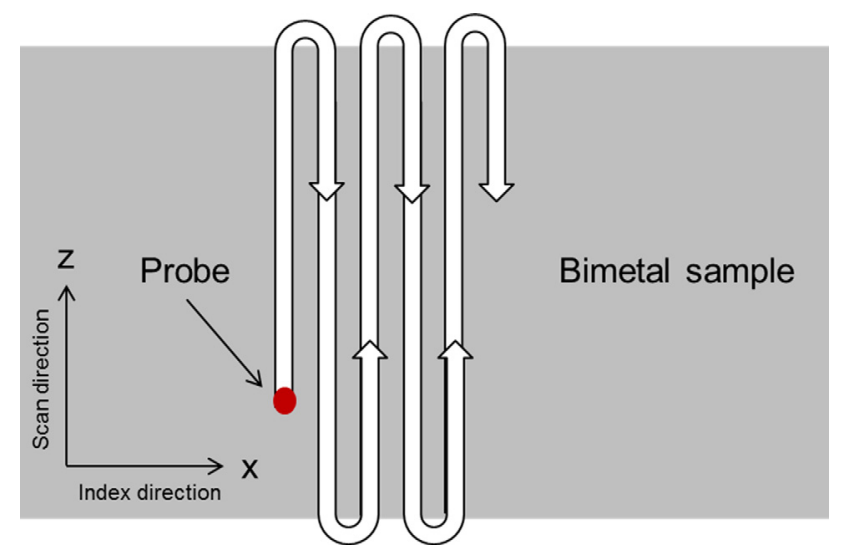

Figure 8. Schematic diagram of sample scanning area in $x-z$ plane of bimetal sample.

under normal conditions and used as reference sample, for which good bond was confirmed with the peel off test, as there was no clad Al peel back. The artificially implanted delamination in the test samples were produced in the shape of an isosceles triangle. The reason for the isosceles triangle shape is that Gao et al. [37] observed a cyclic behaviour of the received ultrasound signal strength in the through transmission mode, depending on the delamination width. Contaminants to create the delamination were $80 \mathrm{~g} / \mathrm{m}^{2}$ gauge paper in the GW-PT sample and ink in the GW-IT sample, which create a clean and precisely delaminated area. This method does not affect the adjacent bonded interface [58]. The size of the delamination in the shape of an isosceles triangle with two sides of equal length is approximately $500 \mathrm{~mm}$ height and $114 \mathrm{~mm}$ width after CRB. The GW-PT sample had a clad $\mathrm{Al}$ peel back of more than $5 \mathrm{~mm}$ that only ended due to fracture of the clad Al, because of the defined gap. This is a bond fail according to the peel off procedure (Sect. 2.1). Sample GW-IT peel back was about $1 \mathrm{~mm}$, which is indicating weakened bond. The samples were $800 \mathrm{~mm}$ long and $171 \mathrm{~mm}$ wide and were made of $0.53 \mathrm{~mm}$ thick clad Al and $1.22 \mathrm{~mm}$ thick steel sheets after $43 \%$ thickness reduction during CRB.

Figure 10a shows the manufacturing of the GW-PT sample and Figure 10b the GW-IT sample immediately before the clad $\mathrm{Al}$ and steel sheets enter the CRB mill.

\subsubsection{Wave propagation analysis}

Phase velocity and group velocity dispersion curves for the Lamb type guided waves were obtained through numerical modelling for the samples with nominal $0.53 \mathrm{~mm}$ alloy and $1.22 \mathrm{~mm}$ steel thickness using the material properties in Table 1. These curves represent possible frequency and phase velocity test mode combinations that are theoretically ideal to detect bond delamination. Based on empirical testing, a test mode was identified for the feasibility study.

\subsubsection{Experimental setup}

Figure 11 shows the guided wave EMAT test setup. The probes shown in Figure 11a were arranged in the through transmission mode with one transmitter and one receiver. Sound was generated on the steel surface. The wave was reflected at the steel and alloy boundaries and propagated along the plate. The samples were clamped on a table as shown in Figure $11 \mathrm{~b}$ to obtain a plain sample surface to prevent sensor lift-offs. The samples were scanned with the two sensors in a uniform motion along the length of the sample. An Innerspec PowerBox $\mathrm{H}$ hand held, battery operated instrument with 
(a)

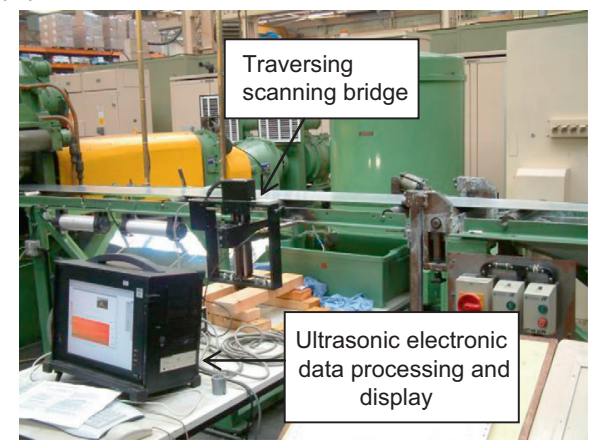

(b)



Figure 9. Experimental setup for online testing: (a) system installed at a position where the strip movement was kept to a minimum and access could be gained to the steel side of the bimetal strip (b) probe and bubbler head.

(a)



(b)

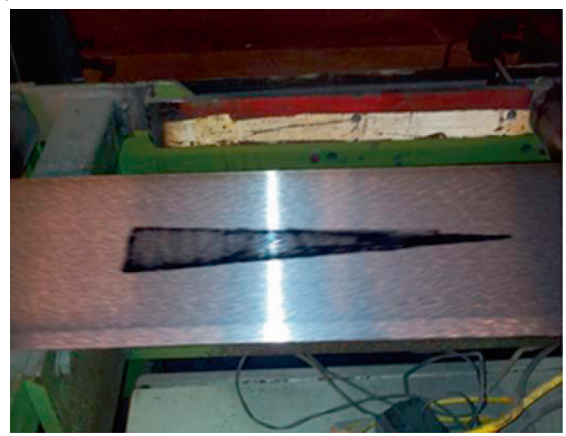

Figure 10. Manufacturing of test samples with isosceles triangle shaped delamination applying (a) $80 \mathrm{~g} / \mathrm{m}^{2}$ gauge paper and (b) ink as contaminant.

(a)



(b)

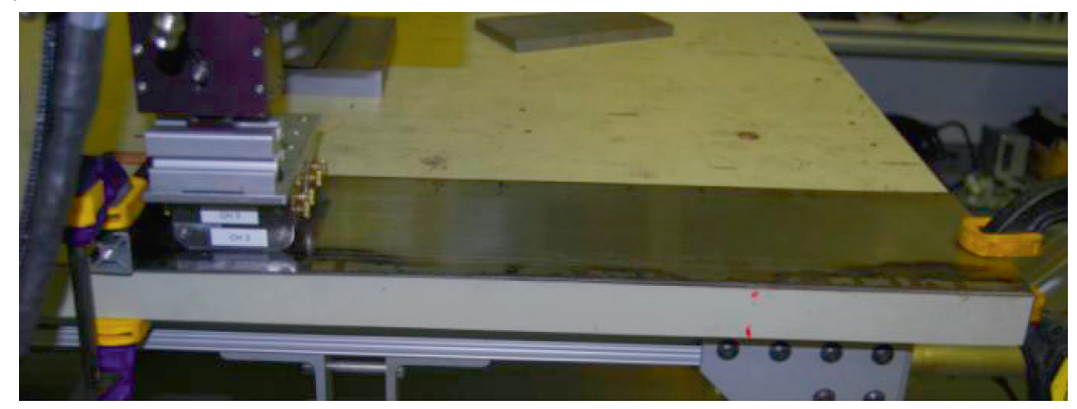

Figure 11. Test setup (a) transmitter and receiver sensors arranged in through transmission mode, (b) sensor setup on test sample.

embedded PowerUT H software was used to process the data and display the information in A, B, C scan formats.

\section{Results}

\subsection{Thermography}

Figure 12a presents active thermography results obtained by inspection of the AT-NL sample. The sample presents a high density of clearly visible small spots with a diameter of few $\mathrm{mm}$, which are likely delamination spots that weaken the bond and reduce the transferable load. These delamination spots were caused by not linishing the steel surface before CRB in the AT-NL sample and reducing the rolling reduction to only $30 \%$ in the AT-RR sample. The darker area is the region that is well bonded, which cooled faster because the heat could flow through the bond. Due to the small size of the delamination spots, the area that was peel off tested comprised multiple delamination spots at the same test sample. The AT-NL and 
(a)

(c)

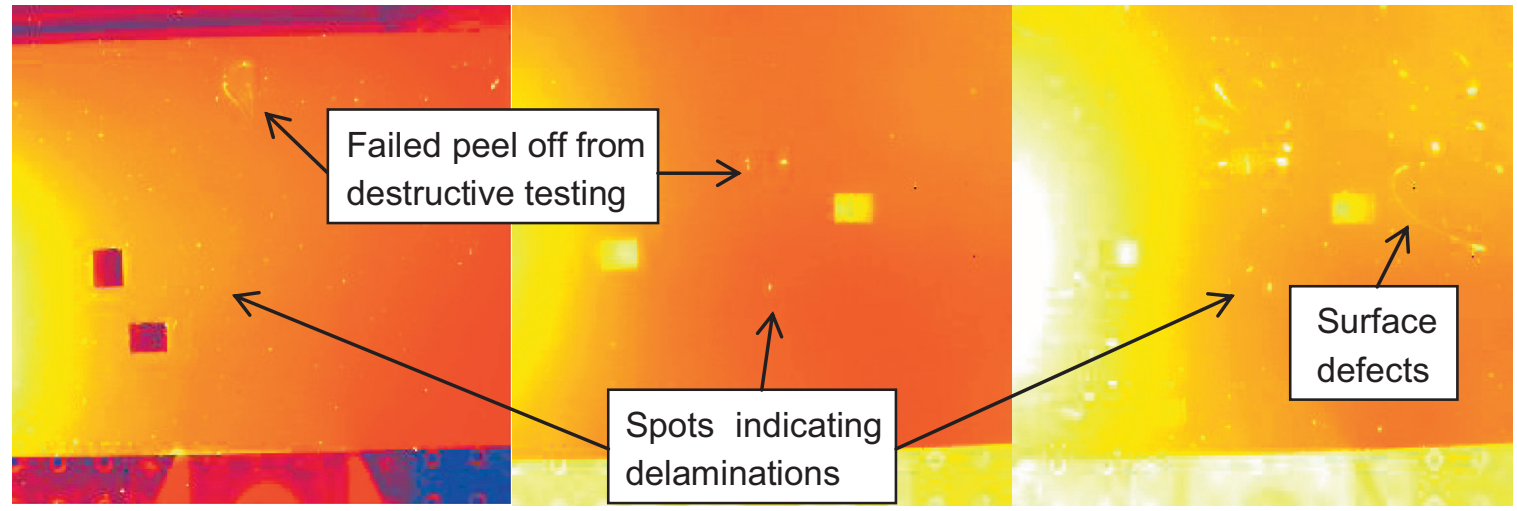

Figure 12. Small spots with a few millimeter diameter present on (a) AT-NL sample, (b) AT-RR sample and (c) AT-RR sample with variation of analysis parameters that revealed surface effects.

AT-RR samples were peel back tested before the thermography inspection. The peel back of the clad Al lining for the AT-NL samples was $13 \mathrm{~mm}$, and for the AT-RR sample $9 \mathrm{~mm}$ respectively, as shown in Figure 12. According to the procedure and acceptability ratings described in Section 2.1, this is a fail test result. Figure $12 \mathrm{~b}$ shows the results for the AT-RR sample with the same analysis parameters like for AT-NL sample. In a direct comparison, the AT-RR sample presents less, but still clearly visible heat spots. Figure $12 \mathrm{c}$ shows thermography results with a variation of analysis parameters for the AT-RR sample, which showed up additional defects on the surface. This is caused by the in-homogenous surface due to indentations and scores, which is usual and within the tolerance for the bimetal strip. Although these surface defects can be countered by adaption of the measurement setup, a continuous system adaption for moving strip is an issue and poses the risk to miss flaws.

Figure 13 shows the AT-R sample without the specific delamination defect structure that is observed on samples AT-NL and AT-RR. The AT-R sample was peel back tested according to the test procedure in Section 2.1 without peel back, which is a pass according to the acceptability criteria. However, it became apparent that the samples have a challenging surface for inspection, which is illustrated in Figure 13. The Al-Sn alloy/steel bimetal sheets feature curvature that can result in reflections depending on the orientation of the two flash lamps and the infrared camera relative to the clad Al surface. The curvature of the bimetal strip results from rolling, annealing and coiling. All test samples that were placed horizontally on the measurement table during inspection did exhibit an upwardly directed curvature. In Figure 13a the normal scale and in Figure 13b the inverted scale is used for inspection.

\subsection{Shearography}

Figure 14 shows the fringe patterns of the S-R and S-SP samples that were tested. Figure $14 \mathrm{a}$ is the fringe pattern of the S-R sample tested with thermal shock loading: as expected the reference sample did not show any visible signs (a)

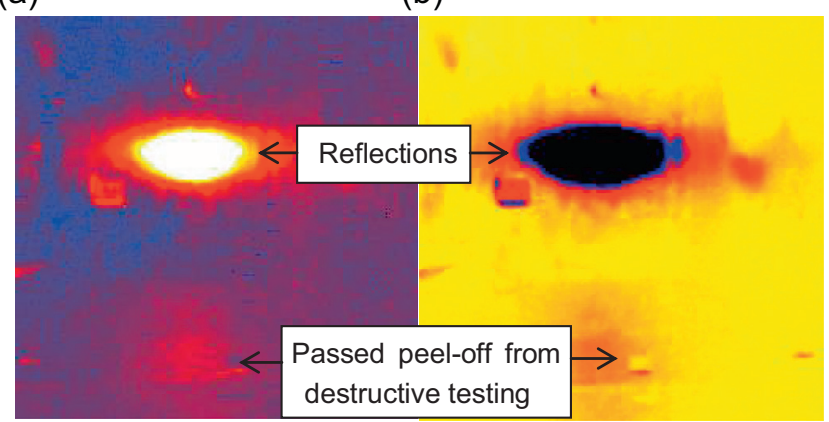

Figure 13. Good parts without specific defect signature but reflections due to curved surface.

of delamination. Figure $14 \mathrm{~b}$ shows the fringe pattern of the S-SP sample under same thermal loading conditions. The delaminated area is clearly visible. It appears as if there were four definable, parallel delaminations within a clear common-defined central $140 \times 45 \mathrm{~mm}$ area through the centre of the sample where the $80 \mathrm{~g} / \mathrm{m}^{2}$ gauge paper was inserted. However, only one piece of $80 \mathrm{~g} / \mathrm{m}^{2}$ gauge paper was inserted.

Figure $14 \mathrm{c}$ shows the fringe pattern of the S-R reference sample that was tested under partial vacuum loading. Again as expected, the S-R sample did not show any visible signs of delamination. Figure $14 \mathrm{~d}$ is the fringe pattern of the S-SP sample under partial vacuum loading, in which the commondefined central $140 \times 45 \mathrm{~mm}$ area through the centre of the sample where the $80 \mathrm{~g} / \mathrm{m}^{2}$ gauge paper was inserted is still clearly visible, but the partial vacuum loading yields a less defined, interconnected delamination area.

\subsection{Ultrasound}

In order to observe the influence of three control factors: sample bond surface preparation, interval between C-scan tests and coupling medium temperature, different levels of these factors were tested. Table 3 summarises the $\mathrm{C}$-scan inspection parameters. The bond surface preparation and temperature 
(a)

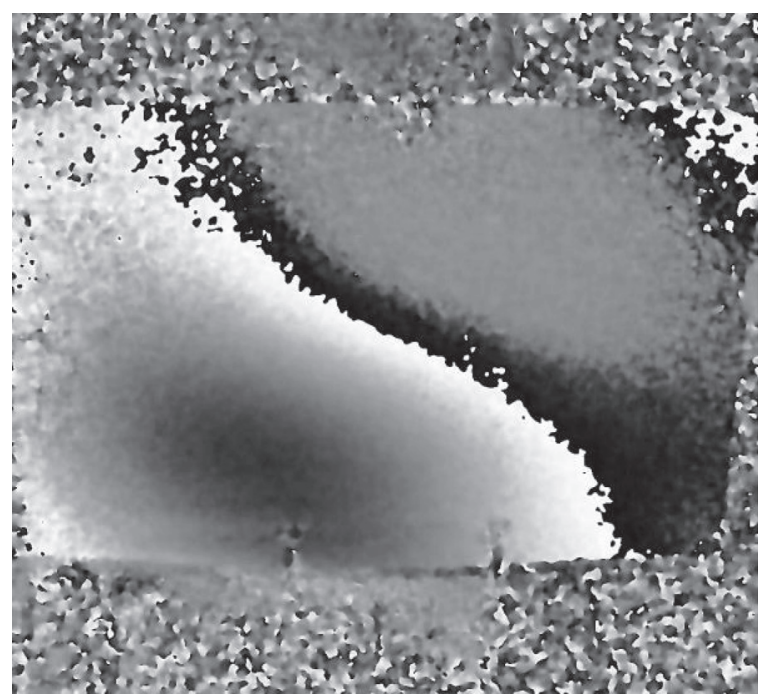

(c)

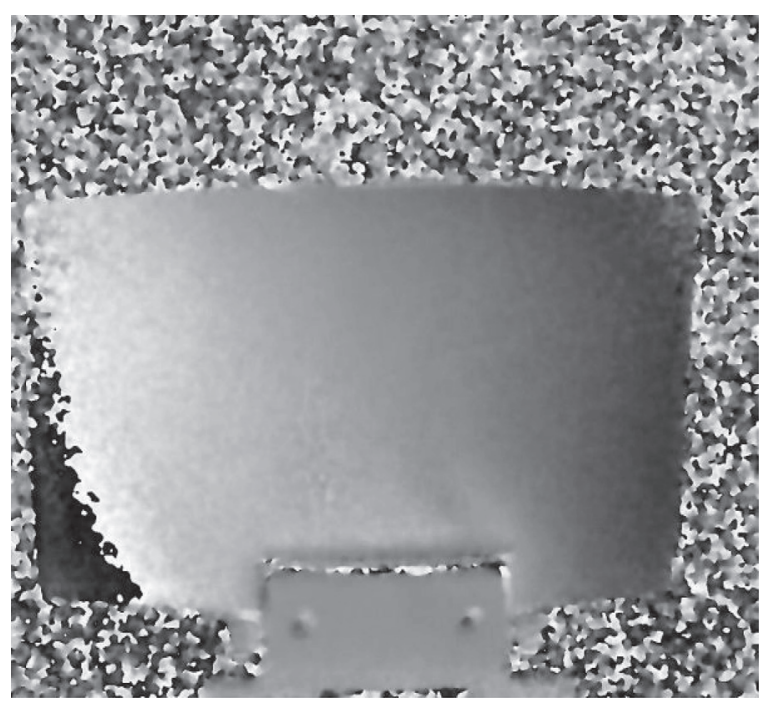

(b)

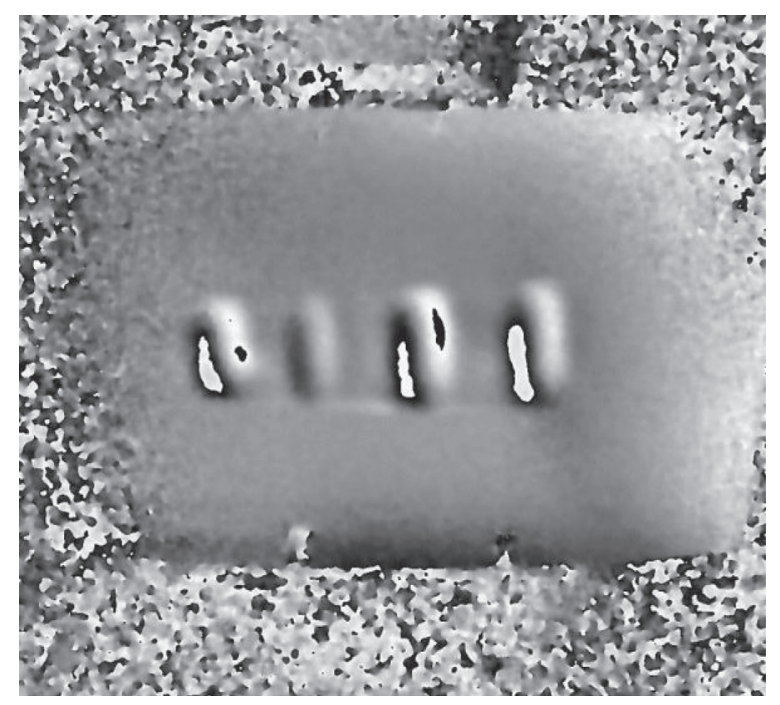

(d)



Figure 14. Image results for thermal testing (a) under normal conditions and (b) with delamination and vacuum testing, (c) under normal conditions and (d) with delamination.

factors have two levels and the interval between the tests has three levels. A two-way analysis of variance (ANOVA) was done for the two different categorical independent variable combinations "surface preparation and interval" as well as "surface preparation and temperature" to examine the influence on the dependent variable ultrasound signal amplitude.

\subsubsection{Influence of time interval between consecutive $\mathrm{C}$-scans}

Figure 15 illustrates maps of the reflected amplitude ratio and its variation across the surface for various samples. This was done to test the time control factor on the C-scan ratio and it became evident that there is a variation in the amplitude ratio with time. Three consecutive tests repeated in a $24 \mathrm{~h}$ interval for three brushed and non-brushed samples were done. The water temperature was kept at $20^{\circ} \mathrm{C}$ during all three days with no alterations to the system. At each time interval,
Table 3. Levels of ultrasound testing factors.

\begin{tabular}{lccc}
\hline \multicolumn{1}{c}{ Control factor } & Level 1 & Level 2 & Level 3 \\
\hline (S) Surface preparation & Brushed & Not brushed & - \\
(I) Interval $(\mathrm{h})$ & 24 & 48 & 72 \\
(T) Temperature $\left({ }^{\circ} \mathrm{C}\right)$ & 20 & 23 & - \\
\hline
\end{tabular}

the brushed samples can be clearly differentiated from the non-brushed samples. After $24 \mathrm{~h}$, the brushed samples show significantly more yellow pixels than red pixels compared to the non-brushed samples. After $48 \mathrm{~h}$, the brushed samples show significantly more blue and less red and yellow pixels than the non-brushed samples. After $72 \mathrm{~h}$, the brushed samples show yet again significantly more blue and almost no more red pixels, compared to the non-brushed samples. 




After $24 \mathrm{~h}$

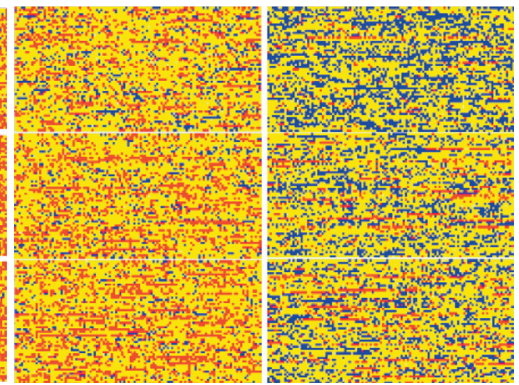

After 48h

Not brushed Brushed

Brushed

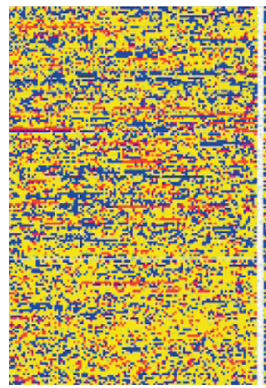

After $72 \mathrm{~h}$

Not brushed



Brushed
1

Figure 15. Three consecutive tests with non-brushed/brushed samples showing a visible difference in the percentage (\%) of red, yellow and blue pixels.

If the non-brushed samples after $48 \mathrm{~h}$ or $72 \mathrm{~h}$ were compared to the brushed samples after $24 \mathrm{~h}$, then the non-brushed samples would appear to have better bond.

Table 4 summarises the statistical analysis of the measurement data. The null hypothesis states that the response means for the main effects of surface preparation and exposure time and their interaction are equal. A significance level of 5\% is considered. The $p$-value for surface preparation for the percentage of red pixels for poor bond and blue pixels for good bond is 0 , which indicates that the surface preparation is associated with the bond strength. The $p$-value for the time interval between the tests is also 0 , indicating that also the interval between the tests is associated with the measured bond strength. Both effects are statistically significant. This confirms that if the non-brushed samples at the $72 \mathrm{~h}$ interval are compared to the brushed samples at the $24 \mathrm{~h}$ interval, the bond quality of the non-brushed samples appears to be better than the brushed samples, although the samples did not physically change. Since the interaction effect between surface preparation and interval is statistically significant, the bond cannot be inspected without considering the interaction effect. It was found that the reason for amplitude variation over time was caused by the water quality. As in serial production inspection conditions it is not practical to consider the interval between tests, the water quality would have to be controlled.

\subsubsection{Influence of temperature on C-scan amplitude ratio}

In oder to test the effect of the water temperature control factor on the C-scan ratio, an extra colour band was introduced to better differentiate between the echo for brushed and nonbrushed material. Values of Gate $1 /$ Gate 2 ratio greater than $110 \%$ are coded red, ratio between 100 and 110 yellow, between 90 and 100 green and ratio below 90 as blue. Figure 16 shows the reflected amplitude ratio map for the non-brushed and brushed samples at $20^{\circ} \mathrm{C}$ and $23{ }^{\circ} \mathrm{C}$. At $20^{\circ} \mathrm{C}$ the samples can be clearly differentiated, as the non-brushed sample shows significantly more red pixels and the brushed sample shows more blue pixels. When the temperature is increased to $23{ }^{\circ} \mathrm{C}$, this significantly affects the received signal and it cannot be differentiated between brushed and non-brushed
Table 4. $p$-Values for two-way ANOVA of surface preparation and exposure time effect.

\begin{tabular}{lcccc}
\hline \multicolumn{1}{c}{ Analysis of variance } & $D F$ & $\%$ Red & \% Yellow & $\%$ Blue \\
\hline Surface preparation & 1 & 0.000 & 0.603 & 0.000 \\
Interval of $24 \mathrm{~h}$ & 2 & 0.000 & 0.000 & 0.000 \\
Surface preparation $\times$ Interval & 2 & 0.049 & 0.000 & 0.001 \\
\hline
\end{tabular}

samples anymore. If the non-brushed sample at $23{ }^{\circ} \mathrm{C}$ were compared to the brushed sample at $20^{\circ} \mathrm{C}$, then the nonbrushed sample would appear to have better bond.

Table 5 summarises the statistical analysis of the measurement data for the temperature influence test. The null hypothesis states that the response means for the main effects surface preparation and water temperature in the tank and their interaction are equal. A significance level of 5\% is considered. The surface preparation $p$-values for the percentage of red, yellow, green and blue pixels are below 0.05 , which indicates that the surface preparation is associated with the bond strength. The temperature $p$-values for the percentage of yellow and green area is below 0.05 , indicating that also the coupling medium temperature is associated with the ultrasound signal. However, for the percentage of red and blue pixels there is no statistical evidence to substantiate this. This could be due to the chosen range of the buckets, which determines if the received Gate 1/Gate 2 amplitude ratio falls either into the category good, weak or unacceptable bond. More trials would be necessary to determine the correct bucket size and back up that they match up with the peel off test results. If the nonbrushed sample results at $23{ }^{\circ} \mathrm{C}$ are compared to the brushed sample results at $20^{\circ} \mathrm{C}$, then the bond quality of the nonbrushed samples appears to be better than for the brushed samples, although the samples did not physically change. Since the interaction effect between surface preparation and temperature is statistically significant, independent of the chosen buckets, the bond cannot be inspected without controlling the coupling medium temperature, which is a challenge considering online inspection in a production environment with environmental influence on the temperature as well as a wide temperature range of the strip itself. 

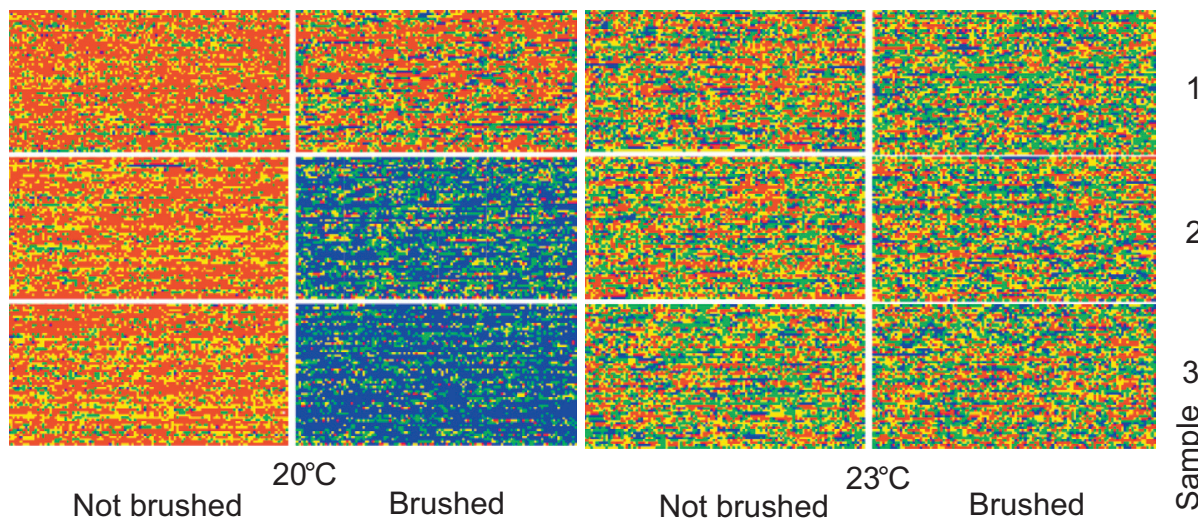

Figure 16. Effect of increasing the water fluid coupling temperature from $20{ }^{\circ} \mathrm{C}$ to $23{ }^{\circ} \mathrm{C}$ for non-brushed and brushed samples.

Table 5. $p$-Values for two-way ANOVA of surface preparation and temperature effect.

\begin{tabular}{lccccc}
\hline \multicolumn{1}{c}{ Analysis of variance } & $D F$ & $\%$ & $\%$ & $\%$ & $\%$ \\
& & Red & Yellow & Green & Blue \\
\hline Surface preparation & 1 & 0.016 & 0.015 & 0.002 & 0.030 \\
Temperature & 1 & 0.191 & 0.034 & 0.000 & 0.342 \\
Surface preparation $\times$ & 1 & 0.037 & 0.038 & 0.045 & 0.046 \\
$\quad$ Temperature & & & & & \\
\hline
\end{tabular}

\subsubsection{C-scan amplitude ratio for samples without known reason for weakened bond}

Samples were taken from serial production that had poor bond, which was detected in the production peel off test. For these samples it was not possible to identify what caused the poor bond. The reflected amplitude ratio map for these samples is shown in Figure 17. All measurements were done at $20^{\circ} \mathrm{C}$ and without delay between each test. Figures $17 \mathrm{a}, 17 \mathrm{~b}$ and $17 \mathrm{~d}$ show reflected amplitude ratio maps of samples that passed the peel off test. Figures $17 \mathrm{c}, 17 \mathrm{e}$ and $17 \mathrm{f}$ are samples that failed the peel off test. It appears that samples that failed the peel off test have less blue pixels and are predominantly yellow and red indicating a poor bond. Samples that that passed the peel off test tend to have more blue pixels indicating a better bond. However, the difference of the reflected amplitude ratio map is too low to differentiate between the samples unambiguously.

Table 6 shows the statistical analysis of the measurement data, which substantiates the qualitative results, that the samples cannot be differentiated based on the reflected amplitude ratio map. A one-way ANOVA was done to compare the percentage of red, yellow and blue pixels on the reflected amplitude ratio map for the production samples. The $p$-value for the percentage of red, yellow and blue pixels is more than 0.05 . This result indicates that the received ultrasound signal for the different samples is not statistically significant. The production samples that failed the bond peel off test cannot be differentiated from samples with good bond with the pulse-echo ultrasound inspection technique.


Figure 17. Samples of variable bond quality (a), (b) and (d) passed the peel off test, (c), (e) and (f) failed the peel off test.

Table 6. $p$-Values for one-way ANOVA of peel off test result.

\begin{tabular}{lcccc}
\hline Analysis of variance & $D F$ & \% Red & \% Yellow & \% Blue \\
\hline Peel off test & 2 & 0.211 & 0.146 & 0.335 \\
\hline
\end{tabular}

\subsection{Guided waves using EMATs}

Figure 18 shows the obtained sample B-scans. For sample GW-R, the received signal amplitude in Figure 18a has only minor variations along the scan. The drop at the end of the scan is where the bimetal sample ended. Figure $18 \mathrm{~b}$ shows the B-scan for the GW-PT sample. The amplitude has a periodic oscillation, which is in accordance with numerical simulation results. Despite the oscillation, the maximum amplitude of the artificial delamination is still significantly below the amplitude for normal condition. Figure 18c shows the B-scan of the GW-IT sample, which has the same characteristics like the GW-PT sample. The test results show that the selected guided wave mode is sensitive to delamination in Al-Sn alloy/steel bimetal strip and can be used for inspection. 
(a)

(b)

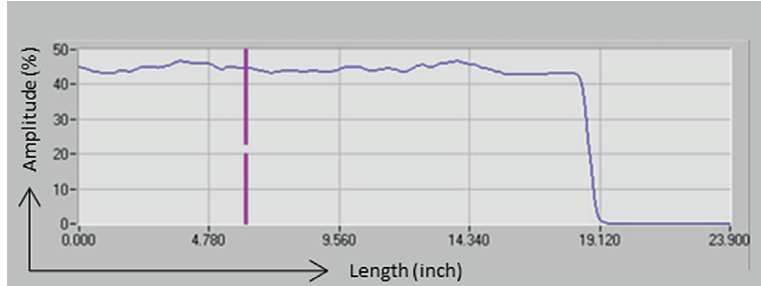

(c)
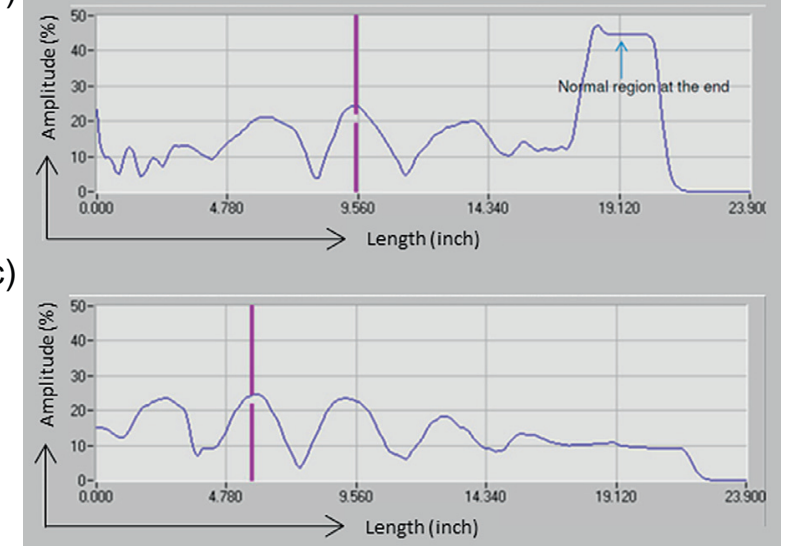

Figure 18. Sample B-scans of (a) control sample, (b) $80 \mathrm{~g} / \mathrm{m}^{2}$ gauge paper delamination sample and (c) ink delamination sample.

\section{Discussion}

Active thermography did detect the artificially implanted poor bond areas. The thermal image showed unambiguously small spots of delaminated area. Since a large area is inspected at the same time, active thermography could keep up with the desired online inspection speed of $12 \mathrm{~m} / \mathrm{min}$. The Al-Sn alloy/steel bimetal samples had a reflective surface, which turned out to be a challenge for active thermography inspection. It is likely that reflections, oil and/or indents that are not affecting the bond, but are present on the strip in serial production, can cause local variations in surface emissivity, which can be misinterpreted as defects. The major limitation for active thermography to be used for online inspection is that the data acquisition of thermography needs to be consistent with the speed of the material loading method, which is challenging considering the varying strip tension, vibration and inspection line speed. Furthermore it is a challenge to reduce the temperature environmental influence in a rough production environment for continuous production, which would be required for online inspection.

The thermal and vacuum shearography testing did clearly detect the artificially implanted delamination. The samples with the artificial flaw showed a clear area of delamination through the centre of the sample and the normal sample showed no visible signs of defect. The surface irregularities of Al-Sn alloy/steel bimetal strip were no issue for inspection with the shearography technique. The maximum inspection area of the used Q- 800 system can reach up to $1.2 \mathrm{~m}^{2}$ within $20 \mathrm{~s}$, which is equivalent to $12 \mathrm{~m} / \mathrm{min}$ for $0.3 \mathrm{~m}$ wide strip. Therewith the feasible inspection speed matches the desired inspection line speed. The major drawback of shearography is the limitation of tolerance to rigid-body motion. This limitation requires loading methods such as thermal or vacuum technique that were used in the laboratory feasibility study. These loading methods are working well under laboratory conditions and for small samples, but their applicability is limited for online inspection due to the varying strip tension and vibrations. This aspect limits the applicability of shearography for the online inspection of Al-Sn alloy/steel bimetal strip, because the movement causes de-correlation of speckle patterns and deterioration of fringe visibility and therewith make flaw detection impossible.

Ultrasound testing is a well-established NDT technique in industry for composite materials and it has been shown that the technique can detect poor bond that is created by not brushing the alloy before cold roll bonding. However, serial production samples that failed the peel off test due to an unknown root cause were not detected. There are practical limitations since the temperature and water quality have to be controlled, because these factors have a significant influence on the measurement result. The same equipment from the laboratory tests was used for online trials to determine the online suitability. During the trials, a series of practical handling issues were encountered. The required water fluid coupling medium was significantly carried over into the bimetal coil, which causes corrosion. Furthermore the strip shape deviations cause difficulties to focus the probe on the bond line whilst the strip is moving. The received ultrasound signal variation with water temperature and quality that was observed in laboratory tests was also observed in the online tests. In regards to $100 \%$ material inspection, a computer controlled scanner can maximise inspection speed and minimise human intervention, hence automation is possible, but scanning of the entire material is still far too slow for $0.3 \mathrm{~m}$ wide strip to keep up with a typical inspection line speed of about $12 \mathrm{~m} / \mathrm{min}$. Furthermore ultrasound requires time consuming and costly calibration, because for each alloy type and alloy/steel thickness combination of the bimetal strip, a calibration sample is required, as the ultrasonic wave velocity is different. In summary, although ultrasonic testing did detect poor bond that was artificially implanted under laboratory conditions, there are many practical issues that obstruct the usefulness of this technique for online inspection of Al-Sn alloy/steel bimetal strips.

Guided waves EMAT is an emerging NDT technique in industry [59] and the non-contact ultrasound generation is the major advantage over conventional ultrasound testing. Based on the set of criteria to be suitable for online inspection and in a direct comparison between all tested NDT techniques, the EMAT technology using guided waves is the most promising technique for inspection of the bond between clad $\mathrm{Al}$ and steel. It uses the advantages of ultrasound testing and overcomes its weaknesses as it does not require a coupling medium and scanning of the material. In an application for inspection of material used to stamp dollar coins, a scanning speed of up to $360 \mathrm{~m} / \mathrm{min}$ for $0.3 \mathrm{~m}$ wide strip was reported [37], which is by far faster than the online inspection speed requirements for Al-Sn alloy/steel bimetal strip. Scanning as required in conventional ultrasound methods is eliminated because for guided waves, the two-dimensional scan is reduced to a one-dimensional scan, which allows $100 \%$ volume inspection 
Table 7. Advantages and limitations of active thermography bond inspection of Al-Sn alloy/steel bimetal strip.

\begin{tabular}{ll}
\hline \multicolumn{1}{c}{ Advantages } & \multicolumn{1}{c}{ Limitations } \\
\hline Full-field inspection. & Potential to miss flaws due to diffuse and/or reflecting surface. \\
Contactless inspection. & Highly skilled and/or experienced operator required. \\
Revealing flaws in seconds. & Signal interpretation can be ambiguous. \\
Information about criticality of flaw. & \\
\hline
\end{tabular}

Table 8. Advantages and limitations of shearography bond inspection of Al-Sn alloy/steel bimetal strip.

\begin{tabular}{ll}
\hline \multicolumn{1}{c}{ Advantages } & \multicolumn{1}{c}{ Limitations } \\
\hline Full-field inspection. & Difficulty to apply mechanical loading without rigid-body movement in-line/limited to laboratory. \\
Contactless inspection. & Potential to miss flaws due to matt and/or reflecting surface. \\
Revealing flaws in seconds. & Highly skilled and/or experienced operator required. \\
Information about criticality of flaw. & Signal interpretation can be ambiguous. \\
\hline
\end{tabular}

Table 9. Advantages and limitations of ultrasound C-scan bond inspection of Al-Sn alloy/steel bimetal strip.

\begin{tabular}{ll}
\hline \multicolumn{1}{c}{ Advantages } & \multicolumn{1}{c}{ Limitations } \\
\hline $\begin{array}{l}\text { Ultrasound directly interrogates and interacts with } \\
\text { delamination. }\end{array}$ & Time consuming and costly scanning required to inspect 100\% of the material. \\
$\begin{array}{l}\text { Well established technique in industry. } \\
\text { No mechanical loading required. }\end{array}$ & $\begin{array}{l}\text { Poor reproducibility and repeatability. } \\
\text { Requirement for coupling media or immersion bath (difficult for in-line continuous } \\
\text { testing). } \\
\text { Slow automated inspection possible. }\end{array}$ \\
& $\begin{array}{l}\text { Significant water/coupling media carry over. } \\
\text { High calibration effort. } \\
\text { Highly skilled and/or experienced operator required. } \\
\text { Difficulties to focus probe when material is moving. }\end{array}$ \\
\hline
\end{tabular}

Table 10. Advantages and limitations of guided waves using EMAT bond inspection of Al-Sn alloy/steel bimetal strip.

\begin{tabular}{ll}
\hline \multicolumn{1}{c}{ Advantages } & \multicolumn{1}{c}{ Limitations } \\
\hline Ultrasound directly interrogates and interacts with delamination. & $\begin{array}{c}\text { Rigid mechanical integration required to avoid any lift offs } \\
\text { between test specimen and probe. } \\
\text { High calibration effort. } \\
\text { High reproducibility and repeatability, not dependent on operator. } \\
\text { No requirement for coupling media or immersion bath. } \\
\text { alloy/steel bimetal strip. }\end{array}$ \\
$\begin{array}{l}\text { Material surface irregularities do not affect the test results. } \\
\text { No mechanical loading required. }\end{array}$ & \\
Low Signal-to-Noise ratio possible. & \\
Fast automated inspection possible. &
\end{tabular}

at high speed. Furthermore EMAT work at elevated temperatures and are insensitive to misalignment [38]. A disadvantage of EMAT is that it requires time consuming and costly calibration like ultrasound testing.

The advantages and limitations of the four NDT techniques tested are summarised in Tables 7-10.

During the laboratory test it was established that in addition to detect the delamination itself, the potential technique needs to be able to cope with specular and matt strip surface, thin oil films up to $5.4 \mathrm{~g} / \mathrm{m}^{2}$ and samples that have surface irregularities such as small indents or scratches.
During the installation of the ultrasound inspection equipment for the online test, the following requirements for online strip inspection were established:

- $100 \%$ of the material must be inspected at an inspection speed of up to $12 \mathrm{~m} / \mathrm{min}$ for strip with a cross section of up to $4.25 \times 300 \mathrm{~mm}$.

- The technique must be able to operate with constantly changing strip tension, which results in vibration and sudden strong movements.

- Automation of the inspection is required. 
Table 11. Feasibility of NDT techniques to fulfil Al-Sn alloy/steel online inspection requirements.

\begin{tabular}{|c|c|c|c|c|c|c|}
\hline $\begin{array}{l}\text { NDT technique/ } \\
\text { criteria }\end{array}$ & $\begin{array}{l}\text { Delamination } \\
\text { detection } \\
\text { capability }\end{array}$ & $\begin{array}{c}\text { Inspection speed } \\
\text { for in-line } \\
\text { application }> \\
12 / \mathrm{min} \\
\end{array}$ & $\begin{array}{c}\text { Compatability with } \\
\text { Al-Sn alloy/steel } \\
\text { bimetal material } \\
\text { properties }\end{array}$ & $\begin{array}{l}\text { Surface irregularities: } \\
\text { scratches, oil, specular } \\
\text { and matt appearance }\end{array}$ & $\begin{array}{l}\text { Strong rigid-body } \\
\text { motion and } \\
\text { vibration }\end{array}$ & $\begin{array}{l}\text { Ambient conditions } \\
\text { changes: light/ } \\
\text { temperature }\end{array}$ \\
\hline Laser shearography & $\checkmark$ & $\checkmark$ & $\checkmark$ & $x$ & $x$ & $x$ \\
\hline Ultrasound & $\checkmark$ & $x$ & $\checkmark$ & $\checkmark$ & $\checkmark$ & $\checkmark$ \\
\hline $\begin{array}{l}\text { Guided wave } \\
\text { EMATs }\end{array}$ & $\checkmark$ & $\checkmark$ & $\checkmark$ & $\checkmark$ & $\checkmark$ & $\checkmark$ \\
\hline
\end{tabular}

- Handling of the equipment must be appropriate in such a way that machine operators can unmistakably distinguish between good and bad material.

- Strip shape deviations that are observed online in the Al-Sn alloy/steel production and that make inspection challenging are coil set, cross bow, torsion, wavy edges, one-sided wavy edges, coil breaks and central buckles.

Table 11 summarises the suitability of the tested NDT techniques for online inspection, based on the established requirements. Among the four tested NDT techniques, EMAT is most suited for online inspection of Al-Sn alloy/steel bimetal strip inspection. The demonstrated measuring sensitivity is adequate for the typical delamination sizes that are expected in Al-Sn alloy/steel bimetal strips. Studies report good repeatability and reproducibility as well as the high inspection speed for $100 \%$ material volume [37]. Furthermore the elimination of the coupling medium leads to the conclusion that guided wave EMAT is a suitable technique for online NDT of Al-Sn alloy/steel bimetal strip.

\section{Conclusions}

A case study of thermography, shearography, ultrasound and guided wave EMAT testing of the bond integrity of Al-Sn alloy/steel bimetal strips is given in this paper. In order to test the feasibility of these techniques to detect delamination, a number of laboratory experiments were done with samples that had different artificially implanted flaws. It was demonstrated that each NDT technique is capable to detect delamination between clad $\mathrm{Al}$ and steel layers under laboratory conditions, but has specific advantages and limitations. The limitations are particularly relevant with regard to online inspection, in which a NDT technique needs to meet more requirements compared to inspecting the bond under laboratory conditions. Active thermography and shearography are both practical enough for inspection under laboratory conditions, but the key issues that prevent an online application are the excitation methods and the environmental influences in the harsh serial production environment, which are not suited for these inspection techniques. Ultrasound testing is well established in industry to inspect the bond of laminates, but these applications are usually for components which are either inspected only point by point, or when it is acceptable that scanning the whole component is very time consuming. Due to the one-dimensional beam and the scanning requirement, the technique is too slow for an online application at elevated speed. Furthermore there are practical issues associated with the requirement for a coupling medium. Guided waves inspection using EMAT was identified as a very promising technique for online NDT of Al-Sn alloy/steel bimetal strip. EMAT has all advantages of ultrasound testing without the limitations that are caused by the conventional ultrasound coupling medium requirement. However, no EMAT inspection system for Al-Sn alloy/steel bimetal strip is currently commercially available.

Acknowledgements. The authors would like to thank MAHLE Engine Systems for the support in providing the test samples and plant resources, DCG Systems for the tests with the active thermography system, Dantec Dynamics for the tests with the shearography system, Physical Acoustics for the loan of the ultrasound system as well as Innerspec Technologies for the simulations and the tests with the EMAT system, which were required for the present study.

\section{References}

1. L. Da Silva, M. El-Sharif, C. Chisholm, S. Laidlaw, A review of the cold roll bonding of AlSn alloy/steel bimetal strips, in: Conference Metal 2014 Proceedings, Brno, 2014.

2. B.-M. Li, J.-F. Han, G.-M. Xu, J.-Z. Cui, Effect of cold-rolling and annealing of interfacial structures and properties of A500/ steel bimetal strip, Transactions of Nonferrous Metals Society of China 15 (2005) 754-758.

3. G.C. Pratt, Materials for plain bearings, International Metallurgical Reviews 18 (1973) 62-88.

4. M. Stolbchenko, O. Grydin, F. Nuernberger, A. Samsonenko, M. Schaper, Sandwich rolling of twin-roll cast aluminium-steel clad strips, in: 11th International Conference on Technology of Plasticity, Nagoya, 2014.

5. R. Jamaati, M.R. Toroghinejad, Investigation of the parameters of the cold roll bonding (CRB) process, Materials Science and Engineering 527 (2010) 2320-2326.

6. T.A. Barnes, I.R. Pashby, Joining techniques for aluminium spaceframes used in automobiles. Part II - adhesive bonding and mechanical fasteners, Journal of Materials Processing Technology 99 (1998) 72-79.

7. B. Drinkwater, P. Cawley, Measurement of the frequency dependence of the ultrasonic reflection coefficient from thin interface layers and partially contacting interfaces, Ultrasonics 35 (1997) 479-488. 
8. R.J. Freemantle, R.E. Challis, Combined compression and shear wave ultrasonic measurements on curing adhesive, Measurement Science and Technology 9 (1998) 1291-1302.

9. S. Dixon, D. Jaques, S.B. Palmer, G. Rowlands, The measurement of shear and compression waves in curing epoxy adhesives using ultrasonic reflection and transmission techniques simultaneously, Measurement Science and Technology 15 (2004) 939-947.

10. E. Siryabe, M. Renier, A. Meziane, M. Castaings, The transmission of lamb waves across adhesively bonded lap joints to evaluate interfacial adhesive properties, in: 2015 ICU International Congress on Ultrasonics, Metz, 2015, 541-544.

11. F. Lanza di Scalea, P. Rizzo, A. Marzani, Propagation of ultrasonic guided waves in lap-shear adhesive joints: case of incident A0 lamb wave, Journal of the Acoustical Society of America 115 (2004) 146-156.

12. R. Seifried, J.J. Laurence, Q. Jianmin, Propagation of guided waves in adhesive bonded components, NDT\&E International 35 (2002) 317-328.

13. Y.Y. Hung, H.P. Ho, Shearography: an optical measurement technique and applications, Materials Science and Engineering: R: Reports 49 (2005) 61-87.

14. M. Hung, Y.S. Chen, S.P. Ng, S.M. Shepard, Y. Hou, J.R. Lhota, Review and comparison of shearography and pulsed thermography for adhesive bond evaluation, Optical Engineering 5 (2007) 051007-051007.

15. Y.Y. Hung, Y.S. Chen, S.P. Ng, L. Liu, Y.H. Huang, B.L. Luk, R.W. Ip, C.M. Wu, P.S. Chung, Review and comparison of shearography and active thermography for nondestructive evaluation, Materials Science and Engineering 64 (2009) 73-112.

16. N.P. Avdelidis, B.C. Hawtin, D.P. Almond, Transient thermography in the assessment of defects of aircraft composites, NDT\&E International 36 (2003) 433-439.

17. N.P. Avdelidis, D.P. Almond, A. Dobbinson, B.C. Hawtin, C. Ibarra-Castanedo, Aircraft composites assessment by means of transient thermal NDT, Progress in Aerospace Sciences 40 (2004) 143-162.

18. C. Meola, G.M. Carlomagno, Application of infrared thermography to adhesion science, Journal of Adhesion Science and Technology 20 (2006) 589-632.

19. S. Bagavathiappan, B.B. Lahiri, T. Saravanan, J. Philip, T. Jayakumar, Infrared thermography for condition monitoring - a review, Infrared Physics \& Technology 60 (2013) 35-55.

20. C. Maierhofer, R. Krankenhagen, R. Roellig, M. Kalisch, J. Meinhardt, Development and application of active thermography for monitoring of deterioration processes of historic structures, in: Nondestructive Testing of Materials and Structures, RILEM Bookseries, 2013, pp. 1111-1116.

21. G. Kim, S. Hong, G.H. Kim, K.Y. Jhang, Evaluation of subsurface defects in fiber glass composite plate using lock-in technique, International Journal of Precision Engineering and Manufacturing 13 (2012) 465-470.

22. B. Yang, P.K. Liaw, M. Morrison, C.T. Liu, R.A. Buchanan, J.Y. Huang, R.C. Kuo, J.G. Huang, D.E. Fielden, Temperature evolution during fatigue damage, Intermetallics 13 (2005) 419-428.

23. M. Omar, M. Hassan, K. Donohue, K. Saito, R. Alloo, Infrared thermography for inspecting the adhesion of plastic welded joints, NDT\&E International 1 (2006) 1-7.

24. S. Marinetti, D. Robba, F. Cernuschi, P.G. Bison, E. Grinzato, Thermographic inspection of TBC coated gas turbine blades:
Discrimination between coating over-thicknesses and adhesion defects, Infrared Physics \& Technology 3 (2007) 281-285.

25. S.M. Shepard, M.F. Beemer, Advances in thermographic signal reconstruction, in: Proceedings of SPIE Thermosense: Thermal Infrared Applications XXXVII, Baltimore, 2015.

26. W. Steinchen, L. Yang, G. Kupfer, P. Maeckel, Non-destructive testing of aerospace composite materials using digital shearography, Journal of Aerospace Engineering 1 (1998) 21-30.

27. N.P. Avdelidis, D.P. Almond, Z.P. Marioli-Riga, A. Dobbinson, B.C. Hawtin, Pulsed thermography: philosophy, qualitative and quantitative analysis on aircraft materials and applications, in: Advances in Signal Processing for Non Destructive Evaluation of Materials, Quebec City, 2005.

28. C. Meola, G.M. Carlomagno, Recent advances in the use of infrared thermography, Measurement Science and Technology 15 (2004) R27.

29. X. Huan, Z. Zeng, W. Li, M. Islam, J. Lu, V. Loggins, E. Yitamben, L.D. Favro, G. Newaz, R.L. Thomas, Acoustic chaos for enhanced detectability of cracks by sonic infrared imaging, Journal of Applied Physics 7 (2004) 3792-3797.

30. F. Chen, Digital shearography: state of the art and some applications, Journal of Electronic Imaging 1 (2001) 240-251.

31. A.V. Fantin, D.P. Willemann, M.E. Benedet, A.G. Albertazzi, Robust method to improve the quality of shearographic phase maps obtained in harsh environments, Applied Optics 55 (2016) 1318-1323.

32. T. Hasiotis, E. Badogiannis, N.G. Tsouvalis, Application of ultrasonic C-scan techniques for tracing defects in laminated composite materials, Journal of Mechanical Engineering 3 (2009) 192-203.

33. B. Djordjevic, Nondestructive test technology for the composites, in: The 10th International Conference of the Slovenian Society for non-destructive testing, Ljubljana, 2009, pp. 259-265.

34. Volume 17: Nondestructive evaluation and quality control, ASM Handbook, 1989.

35. W. Alobaidi, E. Sandgren, H. Al-Rizzo, A survey on benchmark defects encountered in the oil pipe industries, International Journal of Scientific \& Engineering Research 6 (2015) 844-853.

36. C.C. Guyott, P. Cawley, R.D. Adams, The non-destructive testing of adhesively bonded structures: a review, The Journal of Adhesion 20 (1986) 129-159.

37. H. Gao, S. Ali, B. Lopez, Efficient detection of delamination in multilayered structures using ultrasonic guided wave EMATs, NDT\&E International 43 (2010) 316-322.

38. X. Jian, S. Dixon, R.S. Edwards, J. Reed, "Coupling mechanism of electromagnetic acoustical transducers for ultrasonic generation", Journal of the Acoustical Society of America, 119(5), Part 1 (2006) 2693-2701.

39. H.J. Salzburger, F. Niese, G. Dobmann, Emat pipe inspection with guided waves, Welding in the World 56 (2012) 35-43.

40. P.A. Petcher, M.D. Potter, S. Dixon, A new electromagnetic acoustic transducer (EMAT) design for operation on rail, NDT\&E International 65 (2014) 1-7.

41. P.A. Petcher, S. Dixon, Weld defect detection using PPM EMAT generated shear horizontal ultrasound, NDT\&E International 74 (2015) 58-65.

42. K. Arun, R. Dhayalan, K. Balasubramaniam, B. Maxfield, P. Peres, D. Barnoncel, An EMAT-based shear horizontal (SH) wave technique for adhesive bond inspection, in: AIP Conference Proceedings (2012). 
43. S. Huang, Z. Wei, W. Zhao, S. Wang, A new omni-directional EMAT for ultrasonic Lamb wave tomography imaging of metallic plate defects, Sensors 2 (2014) 3458-3476.

44. S. Dixon, C. Edwards, S.B. Palmer, Recent developments in the characterisation of aluminium sheets using electromagnetic acoustic transducers (EMATs), Insight 44 (2002) 274-278.

45. R.S. Edwards, S. Dixon, X. Jian, Characterisation of defects in the railhead using ultrasonic surface waves, NDT\&E International 39 (2006) 468-475.

46. R.S. Edwards, X. Jian, Y. Fan, S. Dixon, Signal enhancement of the in-plane and out-of-plane Rayleigh wave components, Applied Physical Letters 19 (2005) 194104.

47. M. Hirao, H. Ogi, Electromagnetic acoustic resonance and materials characterization, Ultrasonics 6 (1997) 413-421.

48. S. Huang, W. Zhao, Y. Zhang, S. Wang, Study on the lift-off effect of EMAT, Sensors and Actuators S: Physical 2 (2009) 218-221.

49. M. Hirao, H. Ogi, EMATs for science and industry: noncontacting ultrasonic measurements, Kluwer Academic Publishers, London, 2003.

50. R.B. Thompson, Physical principles of measurements with EMAT transducers, vol. 19, Academic Press, Inc., Ames, 1990, pp. 157-200.

51. K. Kawashima, Theory and numerical calculation of the acoustic field produced in metal by an electromagnetic ultrasonic transducer, The Journal of the Acoustical Society of America 60 (1976) 1089-1099.
52. S.B. Palmer, S. Dixon, Industrially viable non-contact ultrasound, Insight 45 (2003) 211-217.

53. H.D. Manesh, H.S. Shahabi, Effective parameters on bonding strength of roll bonded Al/St/Al multilayer strips, Journal of Alloys and Compounds 476 (2009) 292-299.

54. M. Sahin, Effect of surface roughness on weldability in aluminium sheets joined by cold pressure welding, Industrial Lubrication and Tribology 60 (2008) 249-254.

55. R.F. Tylecote, D. Howd, J.E. Furmidge, The influence of surface films on the pressure welding of metals, British Welding Journal 1 (1958) 21-38.

56. M. Movahedi, A.H. Kokabi, S.S. Reihani, Investigation on the bond strength of Al-1100/St-12 roll bonded sheets, optimization and characterization, Materials \& Design 32 (2011) 3143-3149.

57. H.A. Mohamed, J. Washburn, Mechanism of solid state pressure welding, Welding Journal 54 (1975) 302-310.

58. L. DaSilva, M. El-Sharif, C. Chisholm, S. Laidlaw, A novel adaption of the T-peel bimetal bond test based on the thin film bonding theory using cold roll bonded AlSn/steel bimetal laminates, in: Volume 3: Advances in Manufacturing Technology XXX, Loughborough, 2016, 161-166.

59. R.E. Green, Non-contact ultrasonic techniques, Ultrasonics 42 (2004) 9-16.

Cite this article as: Tallafuss PJ, Rosochowski A \& Campbell S: A feasibility study on different NDT techniques used for testing bond quality in cold roll bonded Al-Sn alloy/steel bimetal strips. Manufacturing Rev. 2017, 4, 4. 